\title{
Usefulness of immunohistochemistry for the detection of the BRAF V600E mutation in ovarian serous borderline tumors
}

\author{
YUKO HAYASHI $^{1}$, HIDEFUMI SASAKI ${ }^{2}$, SHO TAKESHITA ${ }^{1}$, RYUTARO NISHIKAWA ${ }^{4}$, \\ HIROSHI NISHIKAWA ${ }^{1}$, ATSUSHI ARAKAWA ${ }^{1}$, YORIKO YAMASHITA ${ }^{3}$, \\ SATORU TAKAHASHI ${ }^{3}$ and MAYUMI SUGIURA-OGASAWARA ${ }^{1}$

\begin{abstract}
Departments of ${ }^{1}$ Obstetrics and Gynecology, ${ }^{2}$ Oncology, Immunology and Surgery, and ${ }^{3}$ Pathology and Tumor Biology, Nagoya City University Graduate School of Medical Sciences, Nagoya 467-8601;

${ }^{4}$ Department of Obstetrics and Gynecology, Nagoya City East Medical Center, Nagoya 464-8547, Japan
\end{abstract}

Received June 24, 2014; Accepted August 1, 2014

DOI: 10.3892/or.2014.3442

\begin{abstract}
Serous borderline tumors and low-grade serous adenocarcinomas typically exhibit a low mitotic index and are largely resistant to chemotherapy. They are characterized by specific mutations, including mutations of KRAS and BRAF, which target specific cell signaling pathways. Mutational analyses may provide further insight into the development sequence of low-grade serous carcinomas. There are 3 methods to detect BRAF mutations: direct sequencing, such as Sanger sequencing (Sas); immunohistochemistry (IHC); and competitive allele-specific hydrolysis probe (TaqMan) PCR technology (CAST-PCR). In the present study, we matched the results of these 3 methods in ovarian serous borderline tumor cases. This study was carried out in 11 surgically removed ovarian serous borderline tumors. Detection of the BRAF V600E mutation was carried out by the FLEX detection system using the VE1 clone antibody and the results were compared with those of Sas and CAST-PCR. The autostainer IHC VE1 assay was positive in 3 of the 11 ovarian serous borderline tumors and negative in the remaining 8 tumors. CAST-PCR demonstrated a BRAF V600E mutation ratio of 16.4, 17.7 and $12.7 \%$, respectively, in the 3 IHC-positive cases. Sas detected the BRAF V600E mutation in only 2 cases, while revealing wild-type BRAF in the remaining 9 cases. Sas revealed KRAS mutations in 2 of these 9 cases with wild-type BRAF. Our data suggest a high concordance rate of the results between CAST-PCR and IHC. Thus, IHC using the VE1 clone and FLEX linker is a specific method for the detection of BRAF V600E and may be an alternative to molecular-biologic techniques for the detection of mutations in ovarian serous
\end{abstract}

Correspondence to: Dr Yuko Hayashi, Department of Obstetrics and Gynecology, Nagoya City University Graduate School of Medical Sciences, 1 Kawasumi, Nagoya 467-8601, Japan

E-mail: yuhayashi0120@hotmail.co.jp

Key words: BRAF mutation, immunohistochemistry, V600E expression, serous borderline tumor, ovary, CAST-PCR borderline tumors. This method may be a useful screening method for the BRAF mutation.

\section{Introduction}

The origin and mechanism of development of ovarian tumors remains unclear. In recent years, a method to classify ovarian cancer into two types, type I and II tumors, has been proposed. Type I tumors comprise low-grade serous adenocarcinoma, low-grade endometrioid adenocarcinoma, clear cell adenocarcinoma and mucinous carcinomas and Brenner tumors. They are generally indolent, present in stage I at diagnosis (tumor confined to the ovary) and characterized by specific mutations, including mutations of KRAS, BRAF and ERBB2, which target specific cell signaling pathways. Type II tumors comprise high-grade serous adenocarcinoma, high-grade endometrioid adenocarcinoma and malignant mixed mesodermal tumors (carcinosarcomas) and undifferentiated carcinomas. They are aggressive, present in an advanced stage at diagnosis and have a very high frequency of TP53 mutations, but rarely harbor the mutations detected in type I tumors (1).

In contrast to patients with high-grade disease, low-grade serous ovarian cancer occurs at a younger age, typically exhibits a low mitotic index and is largely resistant to chemotherapy (2-5). Mutational analyses may provide further insight into the development sequence of low-grade serous carcinomas.

Ovarian serous borderline tumors are relatively rare in Japan and are somewhat more difficult to diagnose. The differentiation of serous borderline tumors from serous adenocarcinoma rests on whether destructive stromal invasion is present or not. According to reports, BRAF gene mutation is detected in $\sim 30-50 \%$ of serous borderline tumors, but in very few serous adenocarcinomas. Furthermore, the reported rate of BRAF or KRAS mutation in serous borderline tumors is $61 \%$ (6), while neither BRAF nor KRAS mutation is found often in invasive high-grade serous adenocarcinomas (6-8). It has recently been shown by whole exome sequencing that low-grade serous ovarian tumors contain very few mutations other than those involving BRAF and KRAS, indicating that MAPK activation is of central importance in the pathogenesis of these neoplasms (9). 
The advent of specific inhibitors of mutant BRAF with clinical activity against malignant melanoma (10) and preclinical data indicating that inhibition of the MAPK pathway in BRAF-mutated ovarian tumors may yield clinical benefit, suggest that reliable and sensitive detection of the V600E mutation may be of clinical relevance in serous ovarian tumors $(11,12)$. Therefore, an accurate and practical assay is urgently needed to detect this molecular subset of ovarian cancer. Currently, the methods available for detecting the mutation are: polymerase chain reaction (PCR)-based assays such as Sanger sequencing (Sas), pyrosequencing, and qPCR. PCR is a single detection test, detecting gene mutations; however, it generally requires multiple tissue sections, dissection for tumor cell enrichment and a multiplex system. Competitive allele-specific TaqMan PCR technology (CAST) for BRAF mutation detection is highly specific and has a sensitivity of $<1 \%$ of mutation rate (13). However, it may require a high quality laboratory infrastructure with welltrained staff and it is also expensive. Recently, it has been demonstrated that mutation-specific antibodies can reliably detect the exchange of even single amino acids in routinely processed, formalin-fixed and paraffin-embedded (FFPE) tumor tissues $(14,15)$. To increase the sensitivity, we modified the BRAF V600E protein expression detection system by immunohistochemistry (IHC) using specific monoclonal antibody, VE1 and Dako EnVision ${ }^{\mathrm{TM}}$ FLEX, using a linker in a Japanese cohort (16). A good concordance of the results has been demonstrated between IHC and direct sequencing $(16,17)$. However, to date, there have been no reported studies that included all three methods, or compared the pros and cons of each. In this study, we matched the results of Sas, IHC and the mutation rate of the BRAF gene detected by CAST-PCR in ovarian serous borderline tumor cases.

\section{Materials and methods}

Patient samples. The study group included 11 patients with ovarian serous borderline tumors who had undergone surgery and were diagnosed as having serous borderline tumor by pathological diagnosis at the Department of Obstetrics and Gynecology, Nagoya City University Hospital, between February 1995 and August 2013. Additionally, 20 randomly selected ovarian cancers, including 6 serous adenocarcinomas, 3 endometrioid adenocarcinomas, 3 clear cell adenocarcinomas, 1 mucinous adenocarcinoma, 2 mucinous borderline tumors, 1 granulosa cell tumor, 1 carcinosarcoma, 1 mixed germ-cell tumor, 1 undifferentiated carcinoma and 1 metastatic colorectal cancer were analyzed by the CAST-PCR BRAF assay. This study was conducted with the approval of the Research Ethics Committee of Nagoya City University Graduate School of Medical Sciences.

VE-1 IHC was previously established in a cohort of 26 lung cancer cases (16), and that study confirmed the presence of BRAF V600E in 5 cases. We used these 5 cases with BRAF V600E and 30 cases of wild-type BRAF, as determined by Sas, as the control samples for CAST-PCR.

Direct sequencing for detecting BRAF and KRAS gene mutations. Genomic DNA was extracted from FFPE tumor tissue using the Qiagen PCR purification kit (Qiagen,
Tokyo, Japan) according to the manufacturer's instructions. DNA concentration was determined with the NanoDrop ND-1000 Spectrophotometer (NanoDrop Technologies Inc., Rockland, DE, USA). The primer sequences for the BRAF gene at exon 15 were: forward primer, 5'-TCATA ATGCTTGCTCTGATAGGA-3' and reverse primer, 5'-GGCC AAAAATTTAATCAGTGGA-3'. The cycling conditions were: initial denaturation at $95^{\circ} \mathrm{C}$ for $3 \mathrm{~min}$, followed by 40 cycles at $95^{\circ} \mathrm{C}$ for $45 \mathrm{sec}, 58^{\circ} \mathrm{C}$ for $45 \mathrm{sec}$ and $72^{\circ} \mathrm{C}$ for $45 \mathrm{sec}$. The PCRs were performed using the rTaq kit (Takara Bio Inc, Shiga, Japan) in a $50 \mu 1$ volume of the reaction mixture. The primer sequences for the KRAS gene were: forward primer, 5'-TCATTATTTTTATTATAAGGCCTGCTGAA-3' and reverse primer, 5'-CAAAGACTGGTCCTGCACCAGTA-3'. The cycling conditions were: initial denaturation at $95^{\circ} \mathrm{C}$ for $3 \mathrm{~min}$, followed by 40 cycles at $94^{\circ} \mathrm{C}$ for $45 \mathrm{sec}, 60^{\circ} \mathrm{C}$ for $45 \mathrm{sec}, 72^{\circ} \mathrm{C}$ for $30 \mathrm{sec}$. The PCR reactions were performed using an LA-Taq kit (Takara Bio Inc.) in a $50 \mu l$ volume of the reaction mixture.

The products were purified using the Qiagen PCR purification kit (Qiagen, Valencia, CA, USA). The amplified cDNAs were separated on $1 \%$ agarose gels and the bands were visualized by ethidium bromide and photographed under ultraviolet transillumination. Sequencing was then carried out with the ABI prism 3100 analyzer (Applied Biosystems Japan Ltd., Tokyo, Japan) and analyzed by BLAST and manual review of chromatograms.

BRAF V600E protein IHC. The 11 tumor specimens were immunostained by automated methods (Dako Japan Co.) for BRAF V600E expression, using the mouse monoclonal BRAF V600E clone, VE1 (Spring Bioscience, Pleasanton, CA, USA). Unstained 4- $\mu \mathrm{m}$ sections of FFPE tumor tissue that were slided from the same FFPE block that were used for extracting genomic DNA were submitted for the analysis. The Dako EnVision $^{\mathrm{TM}}$ FLEX detection system included pretreatment with Dako PT Link (pretreatment module) and Target Retrieval Solution, High pH (K8004, Dako Co., Tokyo, Japan) at $97^{\circ} \mathrm{C}$ for $20 \mathrm{~min}$, followed by incubation with x200 diluted mouse anti-BRAF V600E (clone VE1) with Antibody Diluent (K8006, Dako Co.) overnight at $4^{\circ} \mathrm{C}$. Antibody incubation was followed by standard signal amplification, including with rabbit LINKER (K8019) at room temperature for $15 \mathrm{~min}$, HRP-conjugated EnVision ${ }^{\mathrm{TM}}$ FLEX at room temperature for $20 \mathrm{~min}, \mathrm{DAB}$ reaction for $10 \mathrm{~min}$ and counterstaining with hematoxylin for $3 \mathrm{~min}$. An IHC score was assigned to each case according to the following criteria based on the staining pattern: $3+$, intense, granular cytoplasmic staining; $2+$, moderate, smooth cytoplasmic staining; $1+$, faint cytoplasmic staining; and 0 , no staining. The staining area was categorized as $0-10,25,50,75$ and $90 \%$. Tumors showing positive staining involving a minimum of $50 \%$ of the tumor cells were considered to be positive for BRAF V600E expression. These criteria were determined by one of the co-authors who was blinded to the clinical data.

CAST-PCR. CAST-PCR was carried out using the Mutation Detector $^{\mathrm{TM}}$ (Life Technologies) according to the manufacturer's instructions. CAST is a real-time quantitative Clamp-based PCR technology (qPCR) (18). qPCR allows measurement of a 

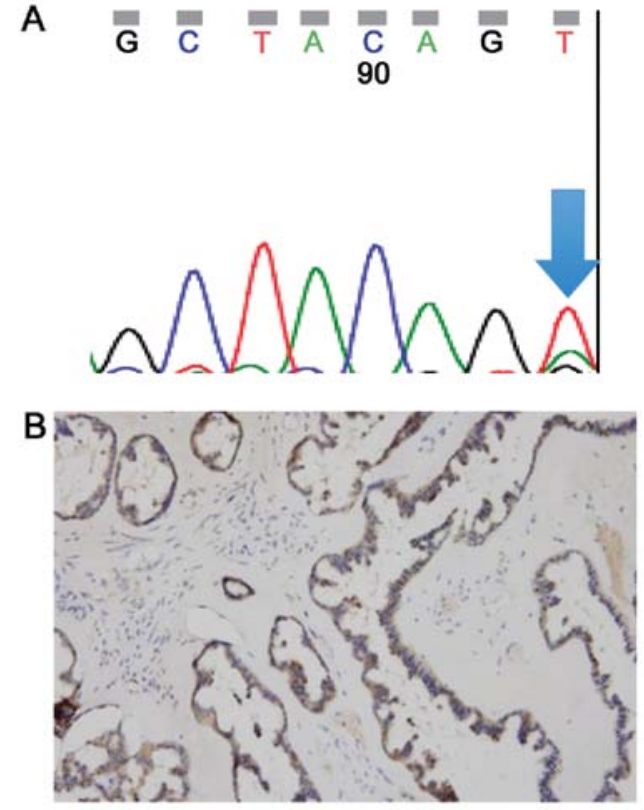

Figure 1. (A) Result of direct sequencing in case 4. There was a change of nucleotide 1799 from thymine to adenine, resulting in a change of the amino acid valine to glutamic acid. (B) Result of IHC in case 4 . This was positive for BRAF mutation; showing 2+ staining intensity with an involved area of $75 \%$.

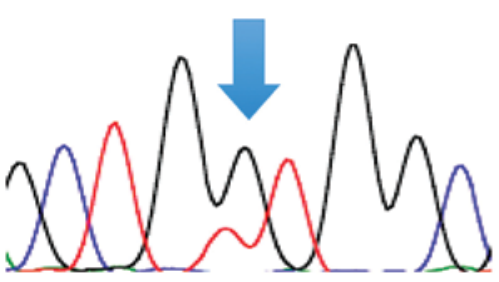

Figure 2. Result of direct sequencing in case 6. KRAS glycine to valine substitution at codon 12 (GGT>GTT, G12V).

quantitative variable, namely, the quantification cycle $(\mathrm{Cq})$ that quantifies the presence of the molecular variant. Each TaqMan mutation detection assay contained a separate set of wild-type and mutant probe mix. qPCRs were run in a final volume of the reaction mixture of $10 \mu \mathrm{l}$ in 96 -well plates containing $5 \mu \mathrm{l}$ of 2x TaqMan genotyping master mix (Life Technologies), $1 \mu \mathrm{l}$ of $10 \mathrm{x}$ assay mix for allele 1 (or 2), $2.5 \mu \mathrm{l}$ of deionized water and $10 \mathrm{ng}$ of the DNA template. Runs were performed on an Applied Biosystems 7500 real-time PCR System. The cycling conditions for the CAST-PCR were: initial denaturation at $95^{\circ} \mathrm{C}$ for $10 \mathrm{~min}$, followed by 5 cycles at $92^{\circ} \mathrm{C}$ for $15 \mathrm{sec}, 58^{\circ} \mathrm{C}$ for $1 \mathrm{~min}$, then 40 cycles at $92^{\circ} \mathrm{C}$ for $15 \mathrm{sec}, 60^{\circ}$ for $1 \mathrm{~min}$. Specific assays for wild-type and mutant BRAF were commercially obtained from Life Technologies. The data were analyzed with the SDS 2.0 software program (13).

\section{Results}

BRAF and KRAS gene alterations in ovarian serous borderline tumors. We carried out sequencing of exon 15 of the
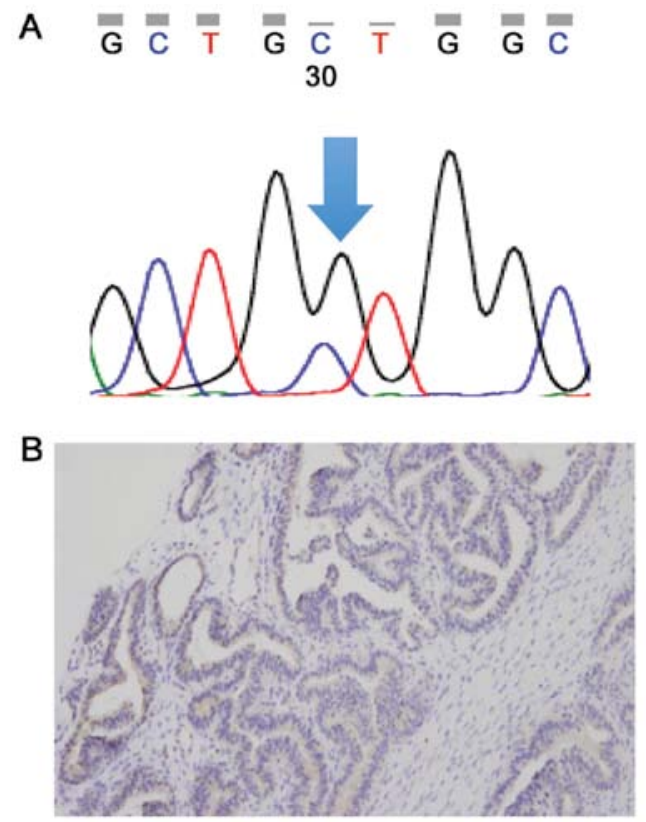

Figure 3. (A) Result of direct sequencing in case 7. KRAS glycine to alanine substitution at codon 12 (GGT>GCT, G12A). (B) Result of IHC in case 7. This showed a 1+ staining intensity with an involved area of $25-50 \%$ and was negative for BRAF mutation by direct sequencing (stained $<50 \%$ ).

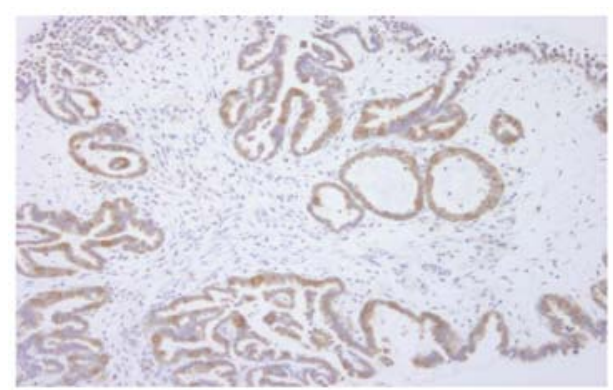

Figure 4. Result of IHC in case 11 . This showed $3+$ staining intensity with an involved area of $90 \%$.

BRAF gene in histopathology specimens of the 11 ovarian serous borderline tumors. Of the 11 tumors, we found 2 cases of BRAF V600E by direct sequencing of DNA samples, which involved a change of nucleotide 1799 from thymine to adenine, resulting in a change of the amino acid valine to glutamic acid (case 4, Fig. 1A). No BRAF mutations were detected in the remaining 9 patients. We also found 2 cases of KRAS mutation among the 11 tumors, consisting of one case of KRAS glycine to valine substitution at codon 12 (GGT>GTT, G12V) (case 6, Fig. 2) and the other of KRAS glycine to alanine substitution at codon 12 (GGT>GCT, G12A) (case 7, Fig. 3A).

Both of the tumors that showed KRAS mutation were BRAF wild-type. Thus, the BRAF and KRAS mutations were mutually exclusive.

IHC for BRAF V600E. Of the 11 tumors, 3 were found by IHC to be positive for BRAF mutation; one of the cases showed $3+$ staining intensity with an involved area of $90 \%$ (case 11 , Fig. 4) and the remaining two showed $2+$ staining intensity with an involved area of $75 \%$ (case 4 , Fig. 1A). The staining 
Table I. Results of IHC, direct sequencing and CAST-PCR.

\section{BRAF}

\begin{tabular}{|c|c|c|c|c|c|}
\hline $\begin{array}{l}\text { Case } \\
\text { (no.) }\end{array}$ & $\begin{array}{c}\text { Age } \\
\text { (years) }\end{array}$ & IHC & $\begin{array}{c}\text { Direct } \\
\text { sequence }\end{array}$ & $\begin{array}{c}\text { CAST-PCR } \\
\text { (mutation rate) }\end{array}$ & $\begin{array}{c}\text { direct } \\
\text { sequence }\end{array}$ \\
\hline 1 & 55 & $\mathrm{~N}$ & $\mathrm{~N}$ & $\mathrm{~N}$ & $\mathrm{~N}$ \\
\hline 2 & 44 & $\mathrm{~N}$ & $\mathrm{~N}$ & $\mathrm{~N}$ & $\mathrm{~N}$ \\
\hline 3 & 40 & $\mathrm{~N}$ & $\mathrm{~N}$ & $\mathrm{~N}$ & $\mathrm{~N}$ \\
\hline 4 & 48 & $\mathrm{P}++$ & $\mathrm{P}$ & $\mathrm{P}(16.3 \%)$ & $\mathrm{N}$ \\
\hline 5 & 51 & $\mathrm{~N}$ & $\mathrm{~N}$ & $\mathrm{~N}$ & $\mathrm{~N}$ \\
\hline 6 & 26 & $\mathrm{~N}$ & $\mathrm{~N}$ & $\mathrm{~N}$ & $\mathrm{P}$ \\
\hline 7 & 55 & $\mathrm{P}$ & $\mathrm{N}$ & $\mathrm{N}$ & $P$ \\
\hline 8 & 27 & $\mathrm{~N}$ & $\mathrm{~N}$ & $\mathrm{~N}$ & $\mathrm{~N}$ \\
\hline 9 & 25 & $\mathrm{P}++$ & $\mathrm{P}$ & $\mathrm{P}(12.7 \%)$ & $\mathrm{N}$ \\
\hline 10 & 65 & $\mathrm{~N}$ & $\mathrm{~N}$ & $\mathrm{~N}$ & $\mathrm{~N}$ \\
\hline 11 & 27 & $\mathrm{P}+++$ & $\mathrm{N}$ & $\mathrm{P}(17.7 \%)$ & $\mathrm{N}$ \\
\hline
\end{tabular}

$\mathrm{P}$, positive; $\mathrm{N}$, negative; IHC, immunohistochemistry.

pattern was diffuse and cytoplasmic. One of the 3 positive cases showed a negative result for BRAF mutation by direct sequencing (case 11).

In addition, one case showed a $1+$ staining intensity with an involved area of $25-50 \%$ (case 7, Fig. 3B) and was negative for BRAF mutation by direct sequencing (stained $<50 \%$ ).

CAST-PCR for BRAF. CAST-PCR using DNA samples from 35 lung cancer cases revealed the BRAF V600E mutation in 5 cases. The mutation ratios were $8,8.9,10,14.9$ and $45.2 \%$, respectively, and all cases were positive by IHC. All 30 cases assessed as wild-type BRAF by Sas were found to be negative by CAST-PCR (data not shown). We used these samples for positive and negative controls.

Of the 11 ovarian serous borderline cases, CAST-PCR carried out using DNA samples revealed BRAF V600E in 3 cases. The mutation ratios were 17.7\% (case 11), 16.3\% (case 4) and $12.7 \%$ (case 9) and all cases were positive by IHC. One case was false-negative by direct sequencing (case 11). All the results are summarized in Table I. The 20 randomly selected ovarian cancers were all wild-type BRAF.

\section{Discussion}

From the results of the detection of the BRAF mutation using 3 methods, one case was false-negative in direct sequencing (case 11). In this study, we used modified methods for the detection of BRAF V600E, namely, EnVision ${ }^{\mathrm{TM}}$ FLEX IHC, a sensitive method using a linker and the results showed excellent concordance of the results with CAST-PCR. As shown by our study as well as previous studies, IHC serves as a highly specific and sensitive test for mutated BRAF and may be used to confirm the diagnosis. The results of BRAF mutation rate in CAST-PCR were not those in the tumor tissues but those of the FFPE graft which we used for extracting DNA. Under controlled conditions, the same FFPE block was used for all 3 methods. When examined with both normal and tumor tissue,
IHC and CAST-PCR were the most effective at detecting BRAF mutations. We also confirmed that IHC was the best of the three methods in terms of accuracy, convenience and economic advantage.

Currently, the presence of point mutations in clinically relevant genes is assessed by various molecular-biologic techniques, including allele-specific PCR, single-strand conformational polymorphism, as well as conventional Sas and pyrosequencing, all of which require a high quality laboratory infrastructure with well-trained staff and are timeconsuming. Currently, Sas is probably the most frequently used technique owing to its reliability and high specificity. However, potential sources of error of molecular-diagnostic techniques include poor DNA quality and an inadequate number of tumor cells in the sample, a relevant problem in the case of low-grade serous ovarian tumors, which was also encountered in our study. The sensitivity rate of Sas generally does not allow for the detection of mutations existing at a frequency of $<15-20 \%$ of tumor cells $(19,20)$. Mutant-specific PCR (MS-PCR) may detect mutations in a greater proportion of cases than routine Sas (21). According to one report, BRAF V600E was detected by Sas in only $32.1 \%$ of melanomas, while $75.9 \%$ of the melanomas had V600E mutations as assessed by MS-PCR (21). Notably, intra- and inter-tumor heterogeneity of BRAF V600E mutations in melanomas has also been reported by the group (21). Amplification refractory mutation system (ARMS)-PCR detected BRAF V600E in only 55\% (30/55) cases of papillary thyroid carcinoma, whereas Sas detected the mutation in 27 of 30 cases (22). We selected CAST-PCR, a technology based on small-fragment amplification and qPCR suitable for low quantities of DNA templates (16). CAST-PCR allows efficient amplification from FFPE samples, the probes are highly specific and all assays have a sensitivity of $<1 \%$ of mutation (16). However, formalin fixation may reduce the DNA quality and potentially affect the performance of the test. The existence of cross reactivity between probes and the consequences of false-positive results need to be evaluated (16).

Recently, mutation-specific antibodies were raised against specific peptide sequences generated by missense mutations $(23,24)$. In particular, BRAF V600E is an ideal target, as it occurs in a broad range of neoplasms and is the most common genetic mutation in human cancers. IHC is a tissue-based, cost-effective technique, which is easy to perform and routinely available in most pathology laboratories (25). Capper et al $(14,15)$ developed a monoclonal mouse antibody (clone VE1) that recognizes the BRAF V600E protein. Several studies have shown that this antibody is reliable for the detection of BRAF V600E in malignant neoplasms by IHC in routinely processed FFPE tissues $(14-16,26)$. VE-1 IHC was previously established in a cohort of 26 Japanese lung cancer cases (16). If the immunohistochemical staining technique is established properly, the sensitivity and specificity may be expected to be equal to those of established molecular-biologic techniques and use of this method may be advantageous for cases with a low percentage of neoplastic cells. A previous report indicated that 22 of 31 serous borderline tumors showed VE-1 IHC positivity, whereas 20 of the 31 tumors were confirmed to show the mutation by allele-specific PCR (17). Two VE1-positive cases with low epithelial cell content required repeat microdissec- 
tion to confirm the presence of the mutation (17). VE-1 IHC positivity was detected in 39 out of 265 colorectal cancer cases (14.7\%), while only 24 of the 39 were confirmed by Sas and only 13 of 15 were confirmed by ultra-deep sequencing (26).

The V600E mutation rate in ovarian serous borderline tumor specimens obtained from the Japanese patients was lower than that in previous Caucasian reports [44.6\% (27) and $30 \%$ (28)], which could be attributed to racial differences in the prevalence of the BRAF mutation. However, V600E substitution accounts for all the BRAF mutations in serous borderline tumors (27). Serous borderline tumors and low-grade serous ovarian cancers are typically chemotherapy-resistant and the reported response rates to cytotoxic chemotherapy are $4 \%$ in the neoadjuvant setting and $2.1-4.9 \%$ in the recurrence setting $(3,4)$. Given the high prevalence of BRAF and KRAS mutations in ovarian serous borderline tumors, testing the effect of inhibitors targeting the MAPK pathway has gained increasing attention. For the case of serous ovarian tumors, preclinical studies demonstrating a profound effect of MAPK pathway inhibition with the compound CI-1040 on tumor cells carrying BRAF or KRAS mutations indicate that this approach may have clinical potential $(11,12)$. The MEK inhibitor selumetinib has been used in a phase II treatment trial for serous ovarian tumors (29).

\section{Acknowledgements}

This study was supported by Grants-in-Aid for Scientific Research, the Japan Society for the Promotion of Science (JSPS) (nos. 24592097 and 25293303). The authors thank Mr. Yoichi Tani, who was responsible for immunostaining.

\section{References}

1. Kurman RJ and Shih IeM: Molecular pathogenesis and extraovarian origin of epithelial ovarian cancer - shifting the paradigm. Hum Pathol 42: 918-931, 2011.

2. Crispens MA, Bodurka D, Deavers M, et al: Response and survival in patients with progressive or recurrent serous ovarian tumors of low malignant potential. Obstet Gynecol 99: 3-10, 2002.

3. Gershenson DM, Sun CC, Bodurka D, et al: Recurrent low-grade serous ovarian carcinoma is relatively chemoresistant. Gynecol Oncol 114: 48-52, 2009

4. Schmeler KM, Sun CC, Bodurka DC, et al: Neoadjuvant chemotherapy for low-grade serous carcinoma of the ovary or peritoneum. Gynecol Oncol 108: 510-514, 2008.

5. Shvartsman HS, Sun CC, Bodurka DC, et al: Comparison of the clinical behavior of newly diagnosed stages II-IV low-grade serous carcinoma of the ovary with that of serous ovarian tumors of low malignant potential that recur as low-grade serous carcinoma. Gynecol Oncol 105: 625-629, 2007.

6. Singer G, Oldt R III, Cohen Y, et al: Mutations in BRAF and KRAS characterize the development of low-grade ovarian serous carcinoma. J Natl Cancer Inst 95: 484-486, 2003.

7. Mayr D, Hirschmann A, Lohrs U and Diebold J: KRAS and BRAF mutations in ovarian tumors: a comprehensive study of invasive carcinomas, borderline tumors and extraovarian implants. Gynecol Oncol 103: 883-887, 2006.

8. Sieben NL, Macropoulos P, Roemen GM, et al: In ovarian neoplasms, BRAF, but not KRAS, mutations are restricted to low-grade serous tumors. J Pathol 202: 336-340, 2004.
9. Jones S, Wang TL, Kurman RJ, et al: Low-grade serous carcinomas of the ovary contain very few point mutations. J Pathol 226: 413-420, 2012.

10. Bollag G, Hirth P, Tsai J, et al: Clinical efficacy of a RAF inhibitor needs broad target blockade in BRAF-mutant melanoma. Nature 467: 596-599, 2010.

11. Nakayama N, Nakayama K, Yeasmin S, et al: KRAS or BRAF mutation status is s useful predictor of sensitivity to MEK inhibition in ovarian cancer. Br J Cancer 99: 2020-2028, 2008.

12. Pohl G, Ho CL, Kurman RJ, et al: Inactivation of the mitogenactivated protein kinase pathway as a potential target-based therapy in ovarian serous tumors with KRAS or BRAF mutations. Cancer Res 65: 1994-2000, 2005.

13. Didelot A, Le Corre D, Luscan A, et al: Competitive allele specific TaqMan PCR for KRAS, BRAF and EGFR mutation detection in clinical formalin fixed paraffin embedded samples. Exp Mol Pathol 92: 275-280, 2012

14. Capper D, Preusser M, Habel A, et al: Assessment of BRAF V600E mutation status by immunohistochemistry with a mutationspecific monoclonal antibody. Acta Neuropathol 122: 11-19, 2011

15. Capper D, Berghoff AS, Magerle M, et al: Immunohistochemical testing of BRAF V600E status in 1,120 tumor tissue samples of patients with brain metastases. Acta Neuropathol 123: 223-233, 2012.

16. Sasaki H, Shimizu S, Tani Y, et al: Usefulness of immunohistochemistry for the detection of the BRAF V600E mutation in Japanese lung adenocarcinoma. Lung Cancer 82: 51-54, 2013.

17. Bösmüller H, Fischer A, Pham DL, et al: Detection of the BRAF V600E mutation in serous ovarian tumors: a comparative analysis of immunohistochemistry with a mutation-specific monoclonal antibody and allele-specific PCR. Hum Pathol 44: 329-335, 2013.

18. Bustin SA, Benes V, Garson JA, et al: The MIQE guidelines: minimum information for publication of quantitative real-time PCR experiments. Clin Chem 55: 611-622, 2009.

19. Lamy A, Blanchard F, Le Pessort F, et al: Metastatic colorectal cancer KRAS genotyping in routine practice: results and pitfalls. Mod Pathol 24: 1090-1100, 2009.

20. Anderson S, Bloom KJ, Vallera DU, et al: Multisite analytic performance studies of a real-time polymerase chain reaction assay for the detection of BRAF V600E mutations in formalin-fixed, paraffin-embedded tissue specimens of malignant melanoma. Arch Pathol Lab Med 136: 1385-1391, 2012.

21. Yancovitz M, Litterman A, Yoon J, et al: Intra- and inter-tumor heterogeneity of BRAF(V600E) mutations in primary and metastatic melanoma. PLos One 7: e29336, 2012.

22. Huang T, Zhuge J and Zhang WW: Sensitive detection of BRAF V600E mutation by Amplification Refractory Mutation System (ARMS)-PCR. Biomark Res 1: 3, 2013.

23. Brevet M, Arcita M and Ladanyi M: Assessment of EGFR mutation status in lung adenocarcinoma by immunohistochemistry using antibodies specific to the two major forms of mutant EGFR. J Mol Diagn 12: 169-176, 2010.

24. Sahm F, Capper D, Meyer J, et al: Immunohistochemical analysis of 1844 human epithelial and haemotopoietic tumours and sarcomas for IDH1R132H mutation. Histopathology 58: 1167-1172, 2011.

25. Raab S: The cost-effectiveness of immunohistochemistry. Arch Pathol Lab Med 124: 1185-1191, 2000.

26. Rossle M, Sigg M, Ruschoff JH, et al: Ultra-deep sequencing confirms immunohistochemistry as a highly sensitive and specific method for detecting BRAF V600E mutations in colorectal carcinoma. Virchows Arch 463: 623-631, 2013.

27. Grisham RN, Iyer G, Garg K, et al: BRAF mutation is associated with early stage disease and improved outcome in patients with low-grade serous ovarian cancer. Cancer 119: 548-554, 2013.

28. Wong KK, Tsang YT, Deavers MT, et al: BRAF mutation is rare in advanced-stage low-grade ovarian serous carcinomas. Am J Pathol 177: 1611-1617, 2010.

29. Farley J, Brady WE, Vathipadiekal V, et al: Selumetinib in women with recurrent low-grade serous carcinoma of the ovary or peritoneum: an open-label, single-arm, phase 2 study. Lancet Oncol 14: 134-140, 2013. 